\title{
A Density-Based Empirical Likelihood Ratio Approach for Goodness-of-fit Tests in Decreasing Densities
}

\author{
Vahid Fakoor $^{1}$, Masoud Ajami ${ }^{2}$, S. M. A. Jahanshahi ${ }^{3, *}$, Ali Shariati ${ }^{1}$ \\ ${ }^{1}$ Department of Statistics, Faculty of Mathematical Sciences, Ferdowsi University, Mashhad, Iran \\ ${ }^{2}$ Department of Statistics, Vali-e-Asr University of Rafsanjan, Rafsanjan, Iran \\ ${ }^{3}$ Department of Statistics, University of Sistan and Baluchestan, Zahedan, Iran
}

\begin{abstract}
In this paper, we propose a test for the null hypothesis that a decreasing density function belongs to a given parametric family of distribution functions against the non-parametric alternative. This method, which is based on an empirical likelihood (EL) ratio statistic, is similar to the test introduced by Vexler and Gurevich [23]. The consistency of the test statistic proposed is derived under the null and alternative hypotheses. A simulation study is conducted to inspect the power of the proposed test under various decreasing alternatives. In each scenario, the critical region of the test is obtained using a Monte Carlo technique. The applicability of the proposed test in practice is demonstrated through a few real data examples.
\end{abstract}

Keywords Decreasing density, Empirical likelihood, Goodness-of-fit test, Grenander estimator, Monte Carlo simulation.

AMS 2010 subject classifications 62G07, 80M31

DOI: $10.19139 /$ soic-2310-5070-707

\section{Introduction}

Suppose that we are interested in estimating a decreasing density function of $f$. We may employ a non-parametric method to estimate the density function of interest under the decreasing constraint. Alternatively, one can postulate a parametric model to estimate $f$ that addresses the monotonicity restriction. In such situations, an appropriate goodness-of-fit test is required to check the applicability of the presumed model. Therefore, the purpose of this article is to propose a suitable test to address this problem. In other words, we aim to test that $f$ belongs to a given parametric family of decreasing density functions against the non-parametric alternative under the decreasing constraint.

One of the first papers on non-parametric density estimation is Grenander [9], in which it is shown that the non-parametric maximum likelihood estimator (NPMLE) of a decreasing density, consisting in an independent and identically distributed (i.i.d.) sample, is the slope of the least concave majorant of the empirical distribution function. Huang and Wellner [11] studied a Grenander-type estimator for a density function and hazard rate under monotone constraint in a right-censoring model.

Neyman [17], which has inspired many other studies, proposed a method to test a parametric null hypothesis against the non-parametric alternative. Another popular approach for the underlying problem is to reject the null hypothesis if an appropriate non-parametric estimator is far enough from the parametric estimator computed under the null hypothesis. For more information about these two approaches and the related references, Durot and Reboul

\footnotetext{
${ }^{*}$ Correspondence to: S. M. A. Jahanshahi (Email: mjahan@math.usb.ac.ir). Department of Statistics, University of Sistan and Baluchestan,
} Zahedan, Iran.

ISSN 2310-5070 (online) ISSN 2311-004X (print)

Copyright (C) 2020 International Academic Press 
[7] provided a comprehensive review. Durot and Tocquet [8] carried out an study on a goodness-of-fit test for a decreasing regression model: the test suggests rejecting the null hypothesis that the monotone regression model belongs to a parametric family against the alternative if the $L_{1}$-distance between the null hypothesis and the Brunk estimator is large enough. Ducharme and Fontez [6] adapted the smooth method of Neyman [17] to test a regression with a positive and increasing mean. Durot and Reboul [7] developed a test for the null hypothesis indicating a real-valued function of interest is a member of a parametric set against the non-parametric alternative within the monotone constraint, say decreasing. They introduced a general model for the test which covers the monotone density, regression and hazard rate with right-censoring models.

Dong and Giles [5] proposed an empirical likelihood ratio-based method for testing the normality. Considering a comprehensive range of alternative distributions, Dong and Giles [5] drawn comparisons between the finite sample performance of the proposed method and those of four other existing tests, indicating better performance of their method. Another approach for testing a parametric null hypothesis against the non-parametric alternative was introduced by Vexler and Gurevich [23], in which a goodness-of-fit (GOF) test was developed through a density-based empirical likelihood ratio. Vexler and Gurevich [23] particularly employed this test for the normal and uniform families of distributions as the null hypotheses, for which it was obtained that the test exhibited great power and preserved the level of significant $(\alpha)$ very good. Vexler et al. [24] applied the same approach to introduce a GOF test for the inverse Gaussian distribution.

There are many other researches in the statistical literature based on EL technique. For example, Ning and Ngunkeng [19] investigated a goodness-of-fit test for the skew-normal distribution. Inspired by the concept of empirical likelihood, Vexler et al. [25] proposed a likelihood ratio-based test for parametric families of distribution functions which provide researcher by an alternative to the parametric likelihood ratio tests. By employing a distribution-free test statistic, Vexler et al. [25] then developed a package in $\mathrm{R}$ that can be used for testing symmetry of data distributions or comparing K-sample distributions. Vexler et al. [26] introduced a new nonparametric likelihood ratio test for independence between two random variables without specifying a restricted set of dependence structures. Using a density-based EL ratio statistic, Vexler et al. [26] obtained that the proposed test is very powerful in detecting a variety of complex dependence structures (e.g. nonlinear and random-effect dependence).

In addition, Safavinejad et al. [22] presented a goodness-of-fit test for the Rayleigh distribution by using sample entropy. Then, the problem of developing an EL ratio-based test for the logistic distribution was investigated by Alizadeh Noughabi [1]. Following this, Safavinejad et al. [21] investigated into the empirical likelihood approach for a goodness-of-fit test for special parametric null hypothesis of the skew-Laplace distribution against against the unknown alternative. A Monte Carlo simulation study was conducted to show that the proposed method preserve the type I error for the skew-Laplace distribution better than the empirical distribution function tests which are based on a measure of discrepancy between the empirical and hypothesized distributions. Marange and Qin [14] introduced an efficient empirical likelihood ratio method for testing the normality hypothesis using moment constraints of the half-normal distribution. It was revealed that this method can also be adapted to test departures from half-normality.

However, there is no study on EL-based test in all the aforementioned papers with the alternative hypothesis subject to the decreasing constraint. By ignoring the monotone assumption, the above tests still can be applied for the situations when the density function of interest is known to be decreasing. However, the information presented by the assumption and the question conditions are not considered. Consequently, an interesting question from theoretical and practical points of view is to derive a novel goodness-of-fit test through empirical likelihood for the hypotheses subject to decreasing constraint. It is anticipated to improve the power and performance of the test by employing some statistical method that is specifically designed for decreasing density functions. There is no GOF test in the current literature presenting an empirical likelihood method by taking the decreasing assumption into account. Accordingly, we will present a new goodness-of-fit test that out performs the existing methods. The better performance of the test proposed is mainly obtained by using an estimator for the density function that reduces the deviation from the true curve.

Now, an interesting question is how to discern when a set of data belongs to a decreasing density. There have been many studies in the literature concerning decreasing densities. Generally, the validity of the monotone assumption 
depends on the researcher prior experiences and information about the study undertaken. It is the framework of study that suggests whether this assumption is valid or not. However, some statistical tools can be applied to inspect the underlying density features. For instance, a descriptive study on data of interest can provide researchers invaluable information about the underlying distribution. Comparing different methods, histogram is one of the most suitable statistical tools for this purpose.

In this paper, we introduce a GOF test for decreasing densities based on the empirical likelihood ratio statistic. Use a methodology similar to Vexler and Gurevich [23], we propose a GOF test for the alternative hypothesis subject to decreasing constraint. The layout of the rest of this paper is as follows: In Section 2, we present the test statistic and study its consistency under the null and alternative hypotheses. The results of the simulation study on critical values and power of the test statistic will be discussed in Section 3. During Section 4, the performance of the proposed test is compared by another existing method for a set of real data.

\section{The test statistics via $\mathbf{E L}$}

Let $X_{1}, \ldots, X_{n}$ be an i.i.d. sample of size $n$ from an unknown density function $f$. For testing the simple hypothesis $H_{0}{ }^{*}: f=f_{0}$ versus the simple alternative $H_{1}: f=f_{1}$, the likelihood ratio test statistic is defined as

$$
L R=\frac{\prod_{i=1}^{n} f_{1}\left(X_{i}\right)}{\prod_{i=1}^{n} f_{0}\left(X_{i}\right)} .
$$

For the simple alternative hypotheses $H_{0}^{*}$ and $H_{1}$, the Neyman-Pearson lemma indicates that the likelihood ratiobased test is the most powerful test when $f_{0}$ and $f_{1}$ both are known.

Utilizing the EL concept to make a GOF test, Vexler and Gurevich [23] proposed a density-based method for an unknown $f_{1}$ and a known $f_{0}$ which may depend on some unknown parameters to test $H_{0}$ versus $H_{1}$. Approximating the Neyman-Pearson test statistic non-parametrically through likelihood ratios, they introduced extensions that possess a great power. In fact, they applied the empirical likelihood method to derive the values of $f_{H_{1}}(\cdot)$ to maximize the numerator of Equation (1) with the constraint of $\int f(x) d x=1$ under the alternative hypothesis. The empirical likelihood method provides researchers with the auxiliary information, the more information comes via the estimation equation, while have not to choose a parametric family for the data. A comprehensive overview of the empirical likelihood method can be found in Owen [20].

Suppose that we wish to test the following parametric null hypothesis versus the non-parametric alternative that $f$ is decreasing.

$$
H_{0}: f \in\left\{f_{\lambda} ; \lambda \in \Lambda\right\},
$$

where $\Lambda \subseteq \mathbb{R}$ is a given set and also, for every $\lambda, f_{\lambda}$ is a known decreasing density function (up to the parameter $\lambda)$ on $[0, \infty)$.

To be more preside, let the alternative hypothesis be

$$
H_{a}: f \in \mathcal{F},
$$

where $\mathcal{F}=\{f: f$ is decreasing $\}$. According to Grenander (1956), since $\mathcal{F}$ includes decreasing densities, for the random sample $X_{1}, \ldots, X_{n}$, in this class there exists a NPMLE for the density function, which is obtained by:

$$
\hat{f}_{n}\left(X_{i}\right)=\max _{i \leq v \leq n} \min _{0 \leq u \leq i-1} \frac{v-u}{n\left(X_{v}-X_{u}\right)},
$$

in which, without loss of generality, $X_{1}, \ldots, X_{n}$ are assumed to be ordered. We call this estimator the Grenander estimator in the rest of this article.

Inspired by (1), applying the NPMLE (2), we derive a new EL ratio version of GOF test for testing $H_{0}$ against $H_{a}$ as

$$
L R_{n}=\frac{\prod_{i=1}^{n} \hat{f}_{n}\left(X_{i}\right)}{\prod_{i=1}^{n} f_{H_{0}}\left(X_{i} ; \hat{\lambda}\right)},
$$


where $\hat{\lambda}$ is the maximum likelihood estimator of the parameter $\lambda$ under $H_{0}$. It is expected that this technique would lead to a GOF test which has better performance in terms of power against other proposed tests in a class of decreasing distributions. To make decision, we define the following critical region, according to which the null hypothesis is rejected if and only if

$$
\log \left(L R_{n}\right)>C_{\alpha}
$$

where $C_{\alpha}$ is a test threshold.

Bear in mind that it is necessary now to find the asymptotic distribution of the proposed test statistic, $L R_{n}$, which consists in Grenander [9] estimator, and the construction of the critical region depends on this distribution. Also, in order to make comparison in simulation study, it will observed that we need to deduce another asymptotic distribution for another test statistic reached via the Vasicek's entropy estimator. But, the asymptotic distributions of these statistics are broadly identified to be analytically difficult. Indeed, it includes the estimates of parameters of the denominator under $H_{0}$ hypothesis, which affects the variance of the test statistics too.

However, similar to the method proposed in the recent literature concerning goodness-of-fit tests, such as Vexler and Gurevich [23], Hall and Welsh [10], Mudholkar and Tian [15, 16] we will not strive to calculate the critical regions analytically for the introduced tests here. Instead, considering the definition of the test statistic $L R_{n}$, we have estimated the values of $C_{\alpha}$ satisfying the following equation through Monte Carlo method.

$$
\sup _{\lambda} P_{H_{0}}\left\{\log \left(L R_{n}\right)>C_{\alpha}\right\}=\alpha .
$$

Table 2 illustrates the Monte Carlo roots of the above equation for different values of $\alpha$ and $n$ based on exponential distribution samples.

\section{Remark 1}

It is of note that the Grenander estimator defined by (2) is distribution-free (see Grenander [9]), and therefore the numerator of $L R_{n}$ does not dependent on the population unknown parameter. Thus, when $\lambda$ is known as $\lambda_{0}$ under $H_{0}$, the distribution of $L R_{n}$ is not a function of an unknown parameter. Consequently, the $L R_{n}$ test is precise and the critical value $\left(C_{\alpha}\right)$ does not depend on an unknown parameter as well.

The weak consistency of the proposed test statistic is presented in the following theorem. For this purpose, we first define

$$
h(x ; \lambda):=\frac{d}{d \lambda} \log f_{H_{0}}(x ; \lambda),
$$

and consider the following assumptions:

1. $E\left(\left|\log f\left(X_{1}\right)\right|\right)<\infty$.

2. Under $H_{0},\left|\hat{\lambda}_{n}-\lambda\right| \stackrel{p}{\rightarrow} 0$ as $n \rightarrow \infty$.

3. Under $H_{1},\left|\hat{\lambda}_{n}-a\right| \stackrel{p}{\rightarrow} 0$ as $n \rightarrow \infty$, where $a$ is a finite term.

4. Under $H_{0}$ and $H_{1}$, there exists a function $g(\cdot)$ such that $|h(x, \eta)| \leq g(x)$, for all $x \in \mathbb{R}, \eta$ in an open interval containing $\lambda$ and $a$, and $E\left(g\left(X_{1}\right)\right)<\infty$

5. Suppose that the true decreasing density $f$ has a bounded support $S$ on which $f(x) \geq \zeta>0$ is satisfied for all $x \in S$. Also assume that $f$ is continuously differentiable and is bounded away from zero on $S$.

\section{Theorem 1}

Assume that assumptions (1) - (5) are satisfied. Then, under $H_{0}$,

$$
\frac{1}{n} \log \left(L R_{n}\right) \stackrel{p}{\rightarrow} 0
$$

as $n \rightarrow \infty$ and, under $H_{1}$,

$$
\frac{1}{n} \log \left(L R_{n}\right) \stackrel{p}{\rightarrow} E\left\{\log \left(\frac{f_{H_{1}}\left(X_{1}\right)}{f_{H 0}\left(X_{1} \mid a\right)}\right)\right\}>0
$$

where $a$ is a finite term. 
Proof

First, we consider the following component of $\frac{1}{n} \log \left(L R_{n}\right)$ :

$$
I_{n}:=\frac{1}{n} \sum_{i=1}^{n} \log \hat{f}_{n}\left(X_{i}\right)
$$

To deal with $I_{n}$, first note that

$$
\begin{aligned}
I_{n} & =\int \log \left(\hat{f}_{n}(x)\right) d F_{n}(x) \\
& =\int \log \left(\hat{f}_{n}(x)\right) d \widehat{F}_{n}(x) \\
& =\int \hat{f}_{n}(x) \log \left(\hat{f}_{n}(x)\right) d x
\end{aligned}
$$

where $\widehat{F}_{n}$ is the least concave majorant of the empirical distribution function $F_{n}$. The equality in (6) follows from the fact that $\log \left(\hat{f}_{n}(x)\right)$ is constant on intervals $(u, v]$, where $u, v$ are successive vertices of $\widehat{F}_{n}$ and the functions $\widehat{F}_{n}$ and $F_{n}$ have equal increments on these intervals. Since $\widehat{F}_{n}$ is absolutely continuous and is equal to the distribution function of $\hat{f}_{n}$, the equality in (7) is reached. Also, the convention $0 \times \infty=0$ could be accepted because $\hat{f}_{n}(x)$ is zero for all $x>X_{(n)}$, where $X_{(n)}$ denotes the largest order statistic of the random variables $X_{1}, \ldots, X_{n}$.

By using Theorem 3 in Nickl [18], for the estimated functional entropy in (7) it is observed that

$$
I_{n} \stackrel{p}{\rightarrow} E_{f}[\log f(X)]=\int f(x) \log (f(x)) d x
$$

Now, the statistic (3) may be presented in the form of

$$
\begin{aligned}
\frac{1}{n} \log \left(L R_{n}\right)= & I_{n}-\frac{1}{n} \sum_{i=1}^{n} \log f_{H_{0}}\left(X_{i} ; \lambda\right) \\
& +\frac{1}{n}\left\{\sum_{i=1}^{n} \log f_{H_{0}}\left(X_{i} ; \lambda\right)-\sum_{i=1}^{n} \log f_{H_{0}}\left(X_{i} ; \hat{\lambda}_{n}\right)\right\} .
\end{aligned}
$$

Given Equation (8), under $H_{0}$ it is revealed that

$$
I_{n} \stackrel{p}{\rightarrow} E_{f_{H_{0}}}\left[\log f_{H_{0}}\left(X_{1}\right)\right]
$$

Considering the weak law of large numbers and Assumption (1), it could be claimed that

$$
\frac{1}{n} \sum_{i=1}^{n} \log f_{H_{0}}\left(X_{i} ; \lambda\right) \stackrel{p}{\rightarrow} E_{f_{H_{0}}}\left[\log f_{H_{0}}\left(X_{1}\right)\right]
$$

as $n \rightarrow \infty$. Now, applying Assumptions (2) and (4), and one-term Taylor expansion, it is obtained that

$$
\frac{1}{n}\left\{\sum_{i=1}^{n} \log f_{H_{0}}\left(X_{i} ; \lambda\right)-\sum_{i=1}^{n} \log f_{H_{0}}\left(X_{i} ; \hat{\lambda}_{n}\right)\right\}=\frac{1}{n} \sum_{i=1}^{n} h\left(X_{i}, \eta_{n}\right)\left(\lambda-\hat{\lambda}_{n}\right) \stackrel{p}{\rightarrow} 0
$$

as $n \rightarrow \infty$, where the value $\eta_{n}$ falls between $\hat{\lambda}_{n}$ and $\lambda$. Thus, under $H_{0}$ and using (8)-(12), we have

$$
\frac{1}{n} \log \left(L R_{n}\right) \stackrel{p}{\rightarrow} 0
$$


Now, under $H_{1}$, it could be deduced that

$$
\frac{1}{n} \log \left(L R_{n}\right)=I_{n}-\frac{1}{n} \sum_{i=1}^{n} \log f_{H_{1}}\left(X_{i}\right)+\frac{1}{n} \sum_{i=1}^{n} \log \frac{f_{H_{1}}\left(X_{i}\right)}{f_{H_{0}}\left(X_{i} ; a\right)}+\frac{1}{n} \sum_{i=1}^{n} \log \frac{f_{H_{0}}\left(X_{i} ; a\right)}{f_{H_{0}}\left(X_{i} ; \hat{\lambda}_{n}\right)} .
$$

Similarly to the proof of the (12), assuming Conditions (1)-(5), we can conclude that

$$
\frac{1}{n} \log \left(L R_{n}\right) \stackrel{p}{\rightarrow} E_{f} \log \left(\frac{f_{H_{1}}\left(X_{1}\right)}{f_{H_{0}}\left(X_{1} ; a\right)}\right)>0
$$

This completes the proof of Theorem 1.

In fact, Theorem 1 shows that the power of the test goes to 1 as $n \rightarrow \infty$ under the alternative hypothesis, that is, the test is consistent.

\section{Simulation study}

Suppose $X_{1}, \ldots, X_{n}$ are i.i.d. random variables from a distribution function $F$ with the corresponding density function $f$ defined over $[0, \infty)$. It is of interest to test the null hypothesis

$$
H_{0}: f(x)=f_{0}(x ; \lambda)=\lambda e^{-\lambda x}
$$

versus

$$
H_{a}: f \in \mathcal{F},
$$

where $\lambda$ could be either specified or unspecified and $\mathcal{F}=\{f: f$ is decreasing $\}$.

To inspect the performance of the proposed test, the nominal level of significance of $\alpha=0.05$ is considered for each simulation scenario during this section. The tests procedure is described in Section 2 and 3.1. The performance of the tests are evaluated in terms of the level of significance and power, and so the corresponding results are reported in Section 3.2 and 3.3, respectively.

\subsection{Tests}

In this subsection, we consider simultaneously the test statistic introduced in (3) and that in Vexler and Gurevich [23], i.e.

$$
G_{n}=\min _{1 \leq m<n^{1-\delta}} \frac{\prod_{i=1}^{n} 2 m /\left\{n\left(X_{(i+m)}-X_{(i-m)}\right)\right\}}{\hat{\lambda}^{n} \exp \left(-\hat{\lambda} \sum_{i=1}^{n} X_{i}\right)},
$$

to test the null hypothesis $H_{0}$ defined in (14), where $X_{(1)}, \ldots, X_{(n)}$ represent the order statistics corresponding to the sample $X_{1}, \ldots, X_{n}$. Note that $X_{(j)}=X_{(1)}$ if $j \leq 1$, and $X_{(j)}=X_{(n)}$ once $j \geq n$. Meanwhile, $\hat{\lambda}$ represents the MLE of $\lambda$ and also $0<\delta<1$. Accordingly, we reject the null hypothesis if, and only if, $\log \left(G_{n}\right)>C_{\alpha}$, where $C_{\alpha}$ is the critical value corresponding to level $\alpha$ obtained by applying (5).

After Vexler and Gurevich [23] proposed the $G_{n}$ test statistic, Vexler et al. [24] and Alizadeh Noughabi [1] revealed that this test statistic has a greater power in comparison to the other GOF tests. Our principal aim is to compare the test statistic proposed in this article $\left(L R_{n}\right)$ with the $G_{n}$ test statistic. For this purpose, we conduct extensive Monte Carlo simulations to inspect the power of the proposed test for various alternatives.

\subsection{Levels of Significance}

To evaluate the performance of the proposed test $L R_{n}$, the empirical levels of significance were calculated based on 10,000 replications of the tests in controlling Type I error with the given nominal level of $\alpha=0.05$. The test statistic were calculated consisting in the various sample sizes of exponential and half normal distributions. In each iteration of exponential distribution, we obtained the sample test statistics by means of (3) and compare them to 
Table 1. Comparing type I error of considered tests for $\alpha=0.05$

\begin{tabular}{|c|c|c|c|c|c|}
\hline Tests & $n=10$ & $n=20$ & $n=30$ & $n=50$ & $n=100$ \\
\hline$L R_{n}$ & 0.045 & 0.046 & 0.045 & 0.052 & 0.051 \\
$G_{n}$ & 0.046 & 0.044 & 0.049 & 0.045 & 0.050 \\
\hline
\end{tabular}

the critical values given in Table 2. It is worth mentioning that we calculated the critical regions for the half normal distribution similarly, however, we do not present these results here owing to space limitations. The percentage of rejecting the null hypothesis was considered as the size of the proposed test.

The simulated results for the exponential distribution are listed in Table 1. According to this table, it was observed that the Type I error of the $L R_{n}$ and $G_{n}$ tests are well controlled. Also, it is revealed that the empirical level of the underlying tests were reasonably close to the nominal value, 0.05 . Therefore, both of the tests had acceptable performances in this regard.

It should be mentioned that we have calculated the Type I error of the $L R_{n}$ and $G_{n}$ tests for the half normal distribution practically as well. Accordingly, compared with Table 1 , it was observed that the $L R_{n}$ and $G_{n}$ tests had absolutely comparable results and the $L R_{n}$ test exhibited even better performance in terms of the Type I error in this case. However, we do not present this result due to space limitations.

\subsection{Power}

In this section, we investigate the empirical power of the tests. As mentioned, since obtaining the exact distribution of the proposed test statistic is complicated, we estimated the quantiles of the distribution of the test statistic $L R_{n}$ using a Monte Carlo simulation technique. For each scenario, we simulated exponential observations of size $n$ and calculated the $L R_{n}$ statistic, then we iterated this process 10, 000 times. Finally, we obtained upper $\alpha$-percentiles for values of $\alpha$ equal to $0.01,0.025,0.05$ and 0.1 . The estimated values of critical regions for exponential distribution are reported in Table 2.

Table 2. Critical values of $L R_{n}$ statistic

\begin{tabular}{|c|c|c|c|c|c|c|c|c|c|c|}
\hline$\alpha n$ & 10 & 15 & 20 & 25 & 30 & 35 & 40 & 50 & 75 & 100 \\
\hline 0.10 & 6.286 & 7.474 & 8.431 & 9.080 & 9.683 & 10.320 & 10.865 & 11.532 & 12.767 & 15.233 \\
0.05 & 7.223 & 8.513 & 9.487 & 10.089 & 10.770 & 11.413 & 11.937 & 12.576 & 13.770 & 15.942 \\
0.025 & 8.203 & 9.427 & 10.464 & 11.109 & 11.685 & 12.398 & 12.999 & 13.519 & 14.824 & 16.519 \\
0.01 & 9.464 & 10.888 & 11.773 & 12.600 & 13.076 & 13.627 & 14.174 & 15.081 & 16.067 & 17.168 \\
\hline
\end{tabular}

Table 3 and Table 4 compare the empirical power of the test proposed and that of Vexler et al. [24] for the null hypotheses that data belongs to exponential distribution and half normal distribution, respectively. All the considered distributions and the corresponding decreasing density functions included in this simulation study are given in the Appendix.

Table 3 indicates that the empirical power of the tests rose by increasing in the sample sizes. In addition, the test consisting in the $L R_{n}$ statistic had greater power than the $G_{n}$-based test except when the alternatives were HL, $\mathrm{HN}(0.5)$ and $\mathrm{GHN}(1,2)$, for which the power of $G_{n}$ test was moderately greater. Furthermore, the results of the tests for $\mathrm{PaI}(2,0,1)$ using two methods were almost comparable. However, in comparison to the $G_{n}$ test, the power of the $L R_{n}$ test were substantially greater for most of the scenarios, especially when the alternatives were $\mathrm{HC}(2)$, DuMouchel(5), KPI(2,2,0.2,2), Reciprocal(2,5) and Weibull(0,1,0.5).

By comparison, it is obtained in Table 4 that the proposed test exhibited implicit superiority over the $G_{n}$ test for all the alternatives when the null hypothesis indicated the data belongs to a half normal distribution. Moreover, it was obtained that the empirical power of the tests climbed as the sample size increased.

In order to check whether or not the tests are robust, we applied both tests for exponential and half normal distribution as the null hypotheses against four other alternatives for which the conditions 3-4 seem to be problematic. 
Table 3. Power of considered tests for $n=10,20$ and $\alpha=0.05$

\begin{tabular}{|l|c|c|c|c|}
\hline$n$ & \multicolumn{2}{|c|}{10} & \multicolumn{2}{c|}{20} \\
\hline Distributions & $L R_{n}$ & $G_{n}$ & $L R_{n}$ & $G_{n}$ \\
\hline DuMouchel(5) & 0.6831 & 0.1918 & 0.8776 & 0.5155 \\
HL & 0.2645 & 0.3743 & 0.4205 & 0.5941 \\
GHL1(0.5) & 0.1134 & 0.1086 & 0.1353 & 0.1299 \\
GHL3(2) & 0.7811 & 0.7794 & 0.8881 & 0.8691 \\
PaI(2,0.1) & 0.9328 & 0.9816 & 0.9999 & 0.9999 \\
PaII(2,1,0.5) & 0.8810 & 0.8068 & 0.9999 & 0.9974 \\
GDP(0,2,1) & 0.8914 & 0.8084 & 0.9999 & 0.9976 \\
GDP(1,2,1) & 0.8440 & 0.3733 & 0.9942 & 0.8758 \\
KPI(2,2,0.2,2) & 0.9104 & 0.3155 & 0.9974 & 0.7389 \\
BurrXII(1,1) & 0.9600 & 0.6797 & 0.9999 & 0.9906 \\
Recip(2,5) & 0.7139 & 0.1332 & 0.9562 & 0.6011 \\
Weibull(0,1,0.5) & 0.5179 & 0.1188 & 0.8448 & 0.5435 \\
Weibull(0.5,1,0.5) & 0.8732 & 0.5705 & 0.9988 & 0.9700 \\
HN(0.5) & 0.1022 & 0.1446 & 0.1432 & 0.2104 \\
GHN(1,2) & 0.7443 & 0.9996 & 0.8564 & 0.9999 \\
\hline
\end{tabular}

Table 4. Power of considered tests for $n=10,20$ and $\alpha=0.05$

\begin{tabular}{|l|c|c|c|c|}
\hline$n$ & \multicolumn{2}{|c|}{10} & \multicolumn{2}{c|}{20} \\
\hline Distributions & $L R_{n}$ & $G_{n}$ & $L R_{n}$ & $G_{n}$ \\
\hline Exp(1) & 0.2345 & 0.0150 & 0.3881 & 0.1050 \\
DuMouchel(5) & 0.9316 & 0.1601 & 0.9902 & 0.4973 \\
HL & 0.4345 & 0.1680 & 0.5491 & 0.2649 \\
GHL1(0.5) & 0.3235 & 0.0273 & 0.3721 & 0.0396 \\
GHL3(2) & 0.6065 & 0.5365 & 0.8160 & 0.8454 \\
PaI(2,0.1) & 0.9674 & 0.8916 & 0.9999 & 0.9998 \\
PaII(2,1,0.5) & 0.9794 & 0.5157 & 0.9999 & 0.9554 \\
GDP(0,2,1) & 0.9999 & 0.4157 & 0.9999 & 0.9042 \\
GDP(1,2,1) & 0.9998 & 0.2252 & 0.9999 & 0.6664 \\
KPI(2,2,0.2,2) & 0.9999 & 0.2072 & 0.9999 & 0.5833 \\
BurrXII(1,1) & 0.9996 & 0.4435 & 0.9999 & 0.9194 \\
Recip $(2,5)$ & 0.9938 & 0.0222 & 0.9998 & 0.1344 \\
Weibull(0,1,0.5) & 0.8672 & 0.1046 & 0.9922 & 0.4737 \\
Weibull(0.5,1,0.5) & 0.9752 & 0.3289 & 0.9999 & 0.8534 \\
GHN(1,2) & 0.5173 & 0.0514 & 0.7275 & 0.0517 \\
\hline
\end{tabular}

Table 5 compares the power of the proposed test with that of $G_{n}$ test for the null hypothesis that data belongs to exponential distribution while Table 6 reveals the results of similar scenarios for the null hypothesis that data satisfies half normal distribution. Two different sample sizes, $n=10$ and $n=20$ were considered for the simulation results reported in these tables. In both Table 5 and 6, it can be observed that the $L R_{n}$ test produced much better results than $G_{n}$ test for all the underlying alternatives.

Broadly speaking, we can conclude that the proposed $L R_{n}$ test had better performance and exhibited superior power in comparison to the $G_{n}$ test. According to this study, the simulation results suggest the benefits of applying the proposed test $\left(L R_{n}\right)$ in practice. Moreover, we conducted a simulation study for other different levels of significance to inspect and compare the power of $L R_{n}$ and $G_{n}$ tests. The simulation results were comparable to the above tables, and thus not only the proposed test indicated a good performance and high power under 
Table 5. Power of considered tests for $n=10,20$ and $\alpha=0.05$

\begin{tabular}{|l|c|c|c|c|}
\hline$n$ & \multicolumn{2}{|c|}{10} & \multicolumn{2}{c|}{20} \\
\hline Distributions & $L R_{n}$ & $G_{n}$ & $L R_{n}$ & $G_{n}$ \\
\hline $\mathrm{HC}(2)$ & 0.4299 & 0.1358 & 0.6722 & 0.3759 \\
MOHC(3.5) & 0.3211 & 0.1453 & 0.5368 & 0.2144 \\
Lomax $(0.5,0.5)$ & 0.8366 & 0.5477 & 0.9820 & 0.7054 \\
Dagum(0.5,1,1) & 0.9472 & 0.7283 & 0.9984 & 0.9896 \\
\hline
\end{tabular}

predetermined different significance levels, but also exhibited superiority over the $L R_{n}$ test. However, we have not presented this results here due to space restrictions.

Table 6. Power of considered tests for $n=10,20$ and $\alpha=0.05$

\begin{tabular}{|l|c|c|c|c|}
\hline$n$ & \multicolumn{2}{|c|}{10} & \multicolumn{2}{c|}{20} \\
\hline Distributions & $L R_{n}$ & $G_{n}$ & $L R_{n}$ & $G_{n}$ \\
\hline $\mathrm{HC}(2)$ & 0.7754 & 0.1223 & 0.9390 & 0.3581 \\
$\operatorname{MOHC}(3.5)$ & 0.6165 & 0.1092 & 0.8562 & 0.3143 \\
Lomax $(0.5,0.5)$ & 0.9642 & 0.5281 & 0.9992 & 0.9082 \\
Dagum $(0.5,1,1)$ & 0.9916 & 0.6991 & 0.9999 & 0.9834 \\
\hline
\end{tabular}

\section{Illustration}

Through three examples, we illustrate how the proposed test can be applied to the GOF test for the exponential and Lomax distributions. The first real example is a set of data reported by Bekker et al. [3], which corresponds to the survival time (in years) of a group of 46 patients receiving chemotherapy treatment alone. The ordered data are: $0.047,0.115,0.121,0.132,0.164,0.197,0.203,0.260,0.282,0.296,0.334,0.395,0.458,0.466,0.501,0.507$, $0.529,0.534,0.540,0.641,0.644,0.696,0.841,0.863,1.099,1.219,1.271,1.326,1.447,1.485,1.553,1.581$, $1.589,2.178,2.343,2.416,2.444,2.825,2.830,3.578,3.658,3.743,3.978,4.003,4.033$.

As it can be seen in Figure 1, the histogram of the survival time is decreasing in a similar manner to the exponential density function. Also, the Vasicek's and Grenander's estimators and corresponding exponential curves have been plotted and added to the histogram. In comparison with Grenander's estimator, one can infer that the Vasicek's estimator considerably fluctuated, whereas the former is much smoother. In this regard, based on Figure 1 , Grenander's estimator is a better estimator for density of the underlying data.

Regarding the test results for the patients data, $G_{n}$ and $L R_{n}$ statistics were responsible for 2.3741 and 11.081 respectively, which are less than the respective critical values of 2.5168 and 12.377 . Accordingly, we do not have any reason to reject the null hypothesis, meaning the data satisfies the exponential distribution.

The second real data set represents the remission time (in months) of a random sample of 128 bladder cancer patients reported in Lee and Wang (2003). The values recorded for these patients are as follows: 0.08, 2.09, 3.48, $4.87,6.94,8.66,13.11,23.63,0.20,2.23,3.52,4.98,6.97,9.02,13.29,0.40,2.26,3.57,5.06,7.09,9.22,13.80$, $25.74,0.50,2.46,3.64,5.09,7.26,9.47,14.24,25.82,0.51,2.54,3.70,5.17,7.28,9.74,14.76,26.31,0.81,2.62$, $3.82,5.32,7.32,10.06,14.77,32.15,2.64,3.88,5.32,7.39,10.34,14.83,34.26,0.90,2.69,4.18,5.34,7.59,10.66$, $15.96,36.66,1.05,2.69,4.23,5.41,7.62,10.75,16.62,43.01,1.19,2.75,4.26,5.41,7.63,17.12,46.12,1.26,2.83$, $4.33,5.49,7.66,11.25,17.14,79.05,1.35,2.87,5.62,7.87,11.64,17.36,1.40,3.02,4.34,5.71,7.93,11.79,18.10$, $1.46,4.40,5.85,8.26,11.98,19.13,1.76,3.25,4.50,6.25,8.37,12.02,2.02,3.31,4.51,6.54,8.53,12.03,20.28$, 2.02, 3.36, 6.76, 12.07, 21.73, 2.07, 3.36, 6.93, 8.65, 12.63, 22.69 .

It can be observed in Figure 2 that the histogram of the remission time is decreasing in a similar manner to the exponential density function. We then added Vasicek's and Grenander's estimators to the histogram. By drawing comparison between the Vasicek's and Grenander's estimators, it is deduced that while the former varied 

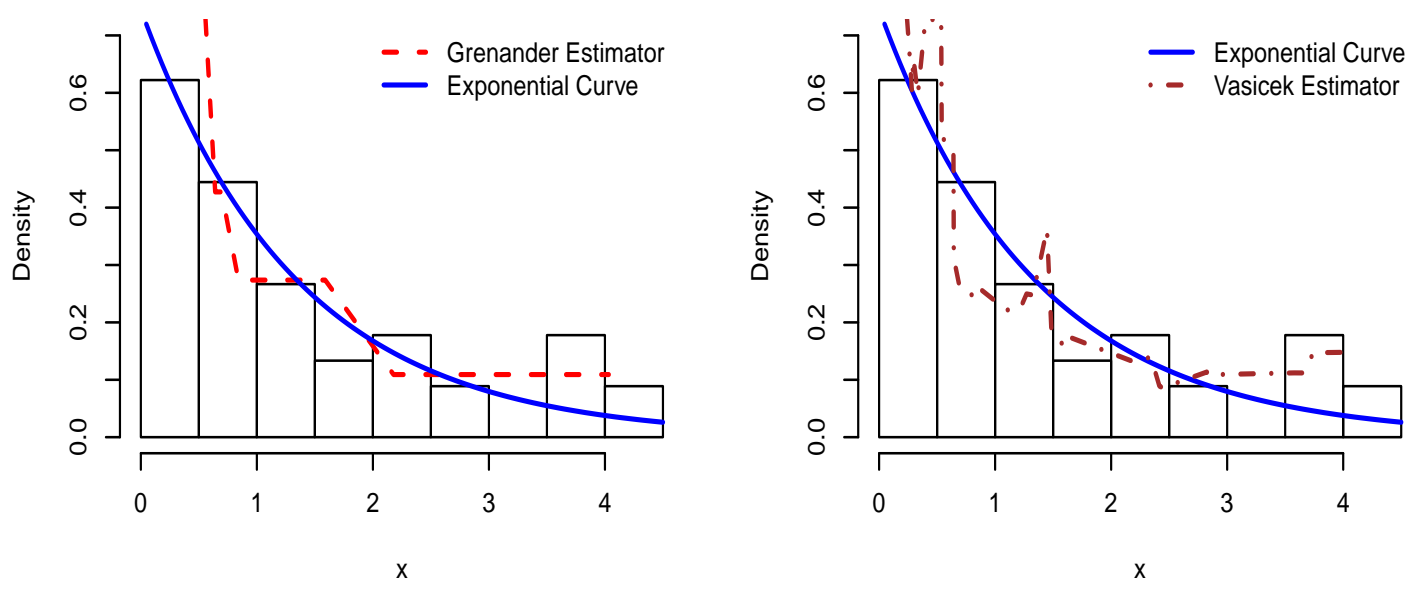

Figure 1. Histogram and Grenander (left panel) and Vasicek (right panel) estimators.
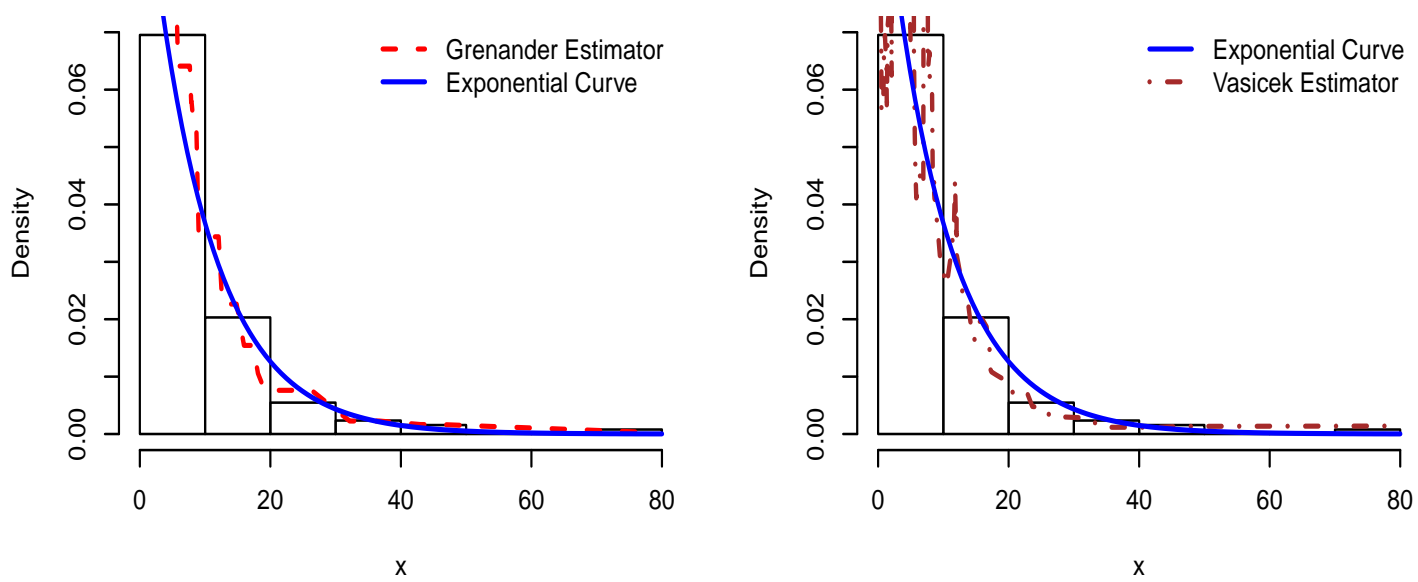

Figure 2. Histogram and Grenander (left panel) and Vasicek (right panel) estimators.

markedly, the latter represented much smoother behavior. Consequently, based upon Figure 2, one can conclude that Grenander's estimator could model the density function of the underlying data better than Vasicek's estimator.

Applying the $G_{n}$ and $L R_{n}$ statistics for the remission data, the respective amounts of 1.6539 and 12.735 were reached, which are less than the corresponding critical values 2.519 and 16.432 , respectively. Thus, the null hypothesis can not be rejected, indicating that the exponential distribution fits adequately.

The third set of data which was given in Hubble [12] includes the distance between extra-galactic nebulae and Earth in mega parsecs. The observations reported in this study included the following values: $0.032,0.034,0.214$, $0.263,0.275,0.275,0.450,0.500,0.500,0.630,0.800,0.900,0.900,0.900,0.900,1.000,1.100,1.100,1.400$, $1.700,2.000,2.000,2.000,2.000$. 

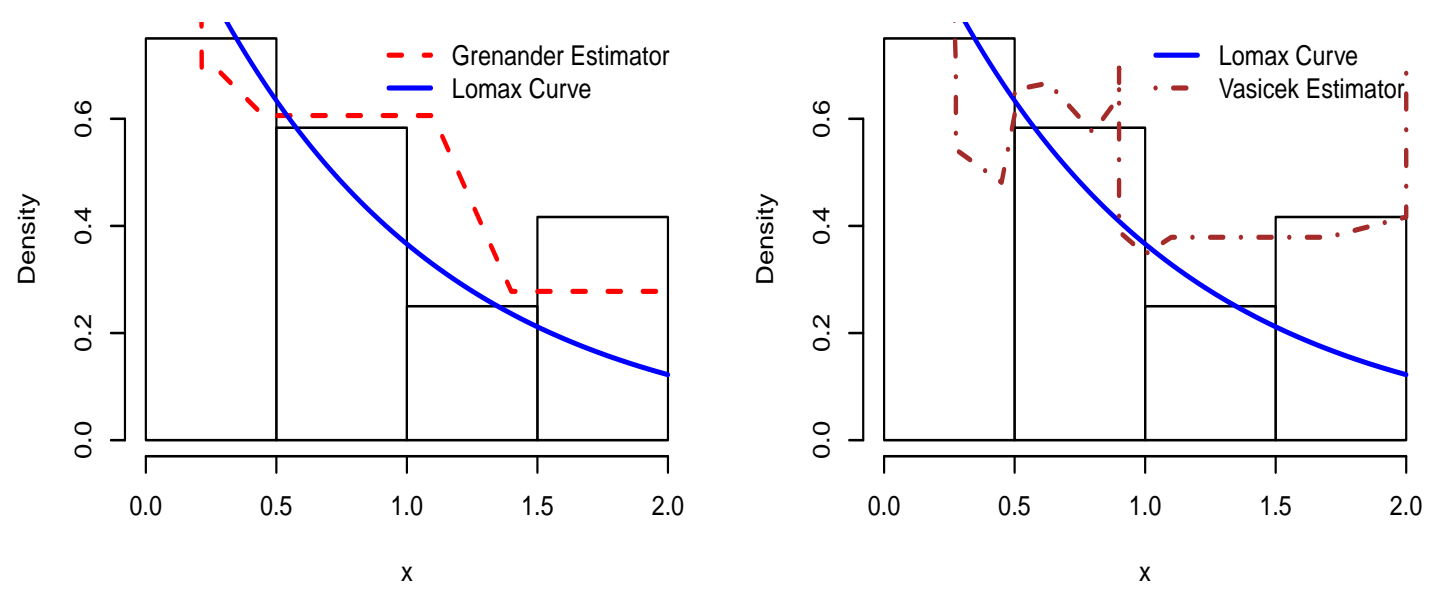

Figure 3. Histogram and Grenander (left panel) and Vasicek (right panel) estimators.

Alshingiti et al. [2] concluded that this set of data follows the Lomax distribution. Figure 3 illustrates the histogram of the distance data as well as the corresponding Vasicek and Grenander estimates. The histogram was observed to follows the Lomax distribution to some degree. This fact alongside with the claim of Alshingiti et al. [2] lead us to consider and plot in Figure 3 the Lomax curve. By contrast with the fluctuations obtained in the Vasicek curve, the Grenander estimator exhibited better results. Compared to the former, the curve for the latter estimates the behavior of data more reasonably.

Turning to the tests' results obtained for the distance data, the $G_{n}$ and $L R_{n}$ statistics accounted for respective 8.200 and 9.639, while the corresponding critical values estimated were equal to 7.987 and 8.543 , respectively. Therefore, the null hypothesis is rejected and, in contrast to the findings of Alshingiti et al. [2], the proposed test indicated that the distance between extra-galactic nebulae and Earth does not satisfy the Lomax distribution using this set of data.

\section{Conclusion}

In this paper, we have presented a test of hypothesis which is specifically designed for decreasing density functions using an empirical likelihood ratio statistic. We have inspected the proposed GOF test for a few distributions, including the exponential and half normal distributions, and have observed that the test outperforms the $G_{n}$ goodness-of-fit test. We have carried out an extensive power comparisons between these tests using a Monte Carlo simulation study. According to the simulation results, we have obtained that in most of the cases for exponential distribution and all the cases for the half normal distribution, the proposed test outperforms its counterpart. Finally, we have presented three sets of real and have illustrated how the proposed test may be applied to evaluate the GOF test for the exponential and Lomax distributions in practice.

\section{Appendix}

The following alternative distributions are considered to obtain power values: 
- Pareto type I distribution $\operatorname{PaI}(\theta, k)$ with the following density function:

$$
f(x)=\frac{\theta k^{\theta}}{x^{\theta+1}}, \quad x \geq k, \theta>0 .
$$

- Pareto type II distribution $\operatorname{PaII}(\alpha, \sigma, \mu)$ with the following density function:

$$
f(x)=\frac{\alpha}{\sigma}\left(1+\frac{x-\mu}{\sigma}\right)^{-(\alpha+1)}, \quad x>\mu, \quad \alpha, \sigma>0 .
$$

- Lomax distribution $\operatorname{Lomax}(\theta, b)$ with the following density function:

$$
f(x)=\frac{\theta b^{\theta}}{(x+b)^{\theta+1}}, \quad x \geq 0, \quad \theta>0 .
$$

- DuMouchel distribution DuMouchel $(\sigma)$ with the following density function:

$$
f(x)=\frac{\sigma}{(x+\sigma)^{2}}, \quad x>0, \sigma>0 .
$$

- Burr XII distribution $\operatorname{BurrXII}(c, k)$ with the following density function:

$$
f(x)=\frac{c k x^{c-1}}{\left(1+x^{c}\right)^{k+1}}, \quad x>0 .
$$

- Inverse Burr distribution $\operatorname{InvBurr}(c, k)$ with the following distribution function:

$$
F(x)=1-\left(1+x^{c}\right)^{-k}, \quad x>0 .
$$

- Half-normal distribution $\mathrm{HN}(\theta)$ with the following density function:

$$
f(x)=\frac{1}{\theta} \sqrt{\frac{2}{\pi}} \exp \left\{-\frac{x^{2}}{2 \theta^{2}}\right\}, \quad x \geq 0, \theta>0 .
$$

- Generalized half-normal distribution $\operatorname{GHN}(\theta, \alpha)$ with the following density function:

$$
f(x)=\sqrt{\frac{2}{\pi}}\left(\frac{\alpha}{x}\right)\left(\frac{x}{\theta}\right)^{\alpha} \exp \left\{-\frac{x^{2 \alpha}}{2 \theta^{2 \alpha}}\right\}, \quad x \geq 0, \theta>0, \alpha>0 .
$$

- Half-logistic distribution HL with the following density function:

$$
f(x)=\frac{2 e^{x}}{\left(1+e^{x}\right)^{2}}, \quad x>0 .
$$

- Type I generalized half-logistic distribution $\operatorname{GHL} 1(\alpha)$ with the following density function:

$$
f(x)=\frac{\alpha}{1+e^{x}}\left(\frac{2 e^{x}}{1+e^{x}}\right)^{\alpha}, \quad x>0, \alpha>0 .
$$

- Type III generalized half-logistic distribution GHL3( $\alpha$ ) with the following density function:

$$
f(x)=\frac{2}{B(\alpha, \alpha)} \frac{e^{\alpha x}}{\left(1+e^{x}\right)^{2 \alpha}}, \quad x>0, \alpha>0 .
$$

- Half-cauchy distribution $\operatorname{HC}(\theta)$ with the following density function:

$$
f(x)=\frac{2}{\pi \theta\left\{1+(x / \theta)^{2}\right\}}, \quad x \geq 0, \theta>0 .
$$


- Marshal-Olkin Half-cauchy distribution $\operatorname{MOHC}(\alpha)$ with the following density function:

$$
f(x)=\frac{2 \pi \alpha}{\left(1+x^{2}\right)\left(\pi \alpha+2(1-\alpha) \tan ^{-1} x\right)^{2}}, \quad x>0, \alpha>0 .
$$

- Reciprocal distribution $\operatorname{Reciprocal}(a, b)$ with the following density function:

$$
f(x)=\left(x \log \frac{b}{a}\right)^{-1}, \quad 0<a \leq x \leq b .
$$

- Weibull distribution Weibull $(a, b, c)$ with the following distribution function:

$$
F(x)=1-\exp \left\{-\left(\frac{x-a}{b}\right)^{c}\right\}, \quad x \geq a .
$$

- Kumaraswamy pareto type I distribution $\operatorname{KPI}(a, b, k, \theta)$ with the following distribution function:

$$
F(x)=1-\left\{1-\left[1-\left(\frac{k}{x}\right)^{\theta}\right]^{a}\right\}^{b}, \quad x \geq k .
$$

- Dagum distribution $\operatorname{Dagum}(a, b, p)$ with the following distribution function:

$$
F(x)=1-\left(1+\left(\frac{x}{b}\right)^{-a}\right)^{-p}, \quad x>0 .
$$

- Generalized pareto distribution $\operatorname{GPD}(\alpha, \beta, \mu)$ with the following distribution function:

$$
F(x)=\left\{\begin{array}{cc}
1-\left(1+\frac{\alpha(x-\mu)}{\beta}\right)^{-1 / \alpha} & \alpha \neq 0 \\
1-\exp \left(-\frac{x-\mu}{\beta}\right) & \alpha=0
\end{array}, \quad x \geq \alpha, \quad \mu \leq x \leq \mu-\beta / \alpha .\right.
$$

\section{Acknowledgement}

We are so grateful to the Editorial Board and to the reviewers for their valuable comments and suggestions which led to this improved version.

\section{REFERENCES}

1. H. Alizaheh, Empirical likelihood ratio-based goodness-of-fit test for the logistic distribution, Journal of Applied Statistics, vol. 42. no. 9, pp. 1973-1983, 2015.

2. A. M. Alshingiti, M. Kayid and H. Aldossary, A new model based on Lomax distribution, International Journal of Reliability and Applications, vol. 15, no. 1, pp. 65-76, 2014.

3. A. Bekker, J. Roux and P. Mostert, A generalization of the compound Rayleigh distribution: using a Bayesian methods on cancer survival times, Communication in Statistics, Theory Methods, vol 29, pp. 1419-1433, 2000.

4. S. G. Donald, G. W. Imbens and W. K. Newey, Empirical likelihood estimation and consistent tests with conditional moment restrictions, Journal of Econometrics, vol. 117, no. 1, pp. 55-93, 2003.

5. L. B. Dong and D. E. A. Giles, An Empirical Likelihood Ratio Test for Normality, Communications in Statistics - Simulation and Computation, vol. 36, no. 1, pp. 197-215, 2007.

6. G. R. Ducharme and B. Fontez, A smooth test of goodness-of-fit for growth curves and monotonic nonlinear regression models, Biometrics, vol 60, no. 2, pp. 977-986, 2004.

7. C. Durot and L. Reboul, Goodness-of-fit test for monotone functions, Scandinavian Journal of Statistics, vol. 37, no. 3, pp. 422-441, 2010.

8. C. Durot and A. S. Tocquet, Goodness-of-fit tests for isotonic regression, ESAIM Probability Statististics, vol. 5, pp. 119-140, 2001.

9. U. Grenander, On the theory of mortality measurement, Part II, Skandinavisk Aktuarietidsskrift, vol. 39, pp. 125-153, 1956.

10. P. Hall and A. H. Welsh, A test for normality based onthe empirical characteristic function, Biometrica, vol. 70, pp. 485-489, 1983. 
11. J. Huang and J. Wellner, Estimation of a monotone density or monotone hazard under random censoring, Scandinavian Journal of Statistics, vol. 22, pp. 3-33, 1995.

12. E. Hubble, A relationship between distance and radial velocity among extragalactic nebulae, newblock Proceedings of the National Academy of Science, pp. 168-179, 1929.

13. E. T. Lee and J. W. Wang, Statistical Methods for Survival Data Analysis, 3rd ed. New York: Wiley. 2003.

14. C. S. Marange and Y. Qin, A Simple Empirical Likelihood Ratio Test for Normality Based on the Moment Constraints of a HalfNormal Distribution, Journal of Probability and Statistics, vol. 2018, pp. 1-10, 2018.

15. G. S. Mudholkar and L. Tian, An entropy characterization of the inverse Gaussian distribution and related goodness-of-fit tests, Journal of Statistical Planning and Inference, vol. 102, pp. 211-221, (2002).

16. G. S. Mudholkar and L. Tian, A test for homogemeity of orderred means of inverse Gaussian population, Journal of Statistical Planning and Inference, vol. 118, pp. 37-49, 2004.

17. J. Neyman, Smooth test for goodness-of-fit, Skandinavisk Aktuarietidsskrift, vol. 20, pp. 149-199, 1937.

18. M. Nickl, Uniform Central Limit Theorems for the Grenander Estimator, Unpublished Manuscript, 2008.

19. W. Ning and G. Ngunkeng, An empirical likelihood ratio based goodness-of-fit test, Statistical Methods and Applications, vol. 22 , no. 2, pp. 209-226, 2013.

20. AB. Owen, Empirical likelihood, New York: Chapman and Hall/CRC, 2001.

21. M. Safavinejad, S. Jomhoori and H. Alizadeh Noughabi, Testing Skew-Laplace Distribution Using Density-based Empirical Likelihood Approach, Journal of Statistical Research of Iran, vol. 13, no. 1, pp. 1-24, 2016.

22. M. Safavinejad, S. Jomhoori and H. Alizade Noughabi, A density-based empirical likelihood ratio goodness-of-fit test for the Rayleigh distribution and power comparison, Journal of Statistical Computation and Simulation, vol. 85, pp. 3322-3334, 2015.

23. A. Vexler and G. Gurevich, Empirical likelihood ratios applied to goodness-of-fit tests based on sample entropy, Computitional Statistics and Data Analysis, vol. 54, pp. 531-545, 2010.

24. A. Vexler, G. G. Shan, S. G. Kim, W. M. Tsai, L. L. Tian and A. D. Hutson, An empirical likelihood ratio based goodness-of-fit test for Inverse Gaussian distributions, Journal of Statistical Planning Inference, vol. 141, pp. 2128-2140, 2011.

25. A. Vexler, H. Tanajian and A. D. Hutson, Density-Based Empirical Likelihood Procedures for Testing Symmetry of Data Distributions and K-Sample Comparisons, The Stata Journal, vol. 14, no. 2, pp. 304-328, 2014.

26. A. Vexler, W. Tsai and A. D. Hutson, A Simple Density-Based Empirical Likelihood Ratio Test for Independence, The American Statistician, vol. 68, no. 3, pp. 158-169, 2014. 